\title{
Current Evidence in Water and Hydration Science
}

\author{
Widjaja Lukito \\ Postgraduate Program in Physician Specialist-I in Clinical Nutrition/Department of Nutrition, Faculty of Medicine, \\ Universitas Indonesia, Jakarta, Indonesia
}

\section{Introduction}

Water constitutes a significant component of the human body. It accounts for $50-80 \%$ of body weight, depending on lean body mass [1]. The human body needs water as an essential medium for metabolism and various biochemical reactions. Many organ functionalities require the role of water. Therefore, water deficit in the forms of hypo- and dehydration leads to organ dysfunction, and, vice versa, many health problems and organ-based diseases contribute to body water imbalance and dysregulation.

Water and hydration are two sides of the same coin. In healthy individuals, water consumption significantly affects hydration status, and, vice versa, hydration status depends on water intake. These relationships are not applicable to overtly sick individuals, e.g., with ascites in liver cirrhosis, edema in nephrotic syndrome, and water retention in hypercortisolism. At this point, water has its functionalities beyond hydration, it has reached out to many dimensions of health and medicine [2]. Water, hydration, and health are three inseparable dimensions that have been comprehensively discussed for 13 years in $\mathrm{Hy}$ dration for Health $(\mathrm{H} 4 \mathrm{H})$ conferences.

The evolution of $\mathrm{H} 4 \mathrm{H}$ conferences has built on its own credibility. $\mathrm{H} 4 \mathrm{H}$ conferences bring together experts from all disciplines with an interest in hydration for health to

karger@karger.com www.karger.com/anm

Karger"
(C) 2022 The Author(s)

Published by S. Karger AG, Basel

This article is licensed under the Creative Commons Attribution 4.0 International License (CC BY) (http://www.karger.com/Services/ OpenAccessLicense). Usage, derivative works and distribution are permitted provided that proper credit is given to the author and the original publisher. share cutting-edge research in this area. $\mathrm{H} 4 \mathrm{H}$ started with a small, invited audience from a narrower range of disciplines and now involves more diverse experts in health, social, and environmental sciences. The 13th edition of $\mathrm{H} 4 \mathrm{H}$ conferences presented, among other themes, emerging evidence on the potential roles of hydration on microbiome and immunity. The active participation of Early Career Researchers demonstrated a sustained capacity of professionals in water and hydration science. Overall, the event sustains more professionals and experts and young professionals and researchers.

The elaboration of the articles in the current proceedings can be at least clustered into five themes: thirst, physiology of hydration and aging; epidemiology of fluid intake patterns; psychology of thirst and hydration; hydration, metabolic responses, and kidney disease; and water, hydration, immune response, and gut microbiota.

\section{Thirst, Physiology of Hydration, and Aging}

Armstrong and Kavouras [3] have reviewed comprehensively the complexity of the thirst paradigm and the drive to drink water in 2019. In short, the understanding of thirst has undergone a long evolution, which has also involved various experts from different disciplines. Ad- 
vances in neuroscience with its imaging technologies have made a significant leap in explaining the phenomenon of thirst and motivation to drink water. In the present proceedings, Bichet [4] affirmed that two brain regions have already been defined to be important in drinking behaviors in animals, the subfornical organ and the organum vasculosum of the lamina terminalis, which can perceive two modes of thirst, osmotic and hypovolemic. Moreover, the peripheral sensory systems in the tongue, which have multimodal sensations, like taste and olfaction, can also taste water through the change in the $\mathrm{pH}$ of the saliva due to dilution by water.

Carroll [5] described four compartment-multidisciplinary models of thirst beyond a "conventional" physiological understanding of thirst, namely true thirst, which is primarily osmoregulated; contextual thirst like that induced by mouth-breathing; pharmacological thirst, as a consequence of drugs and excipients; and impulsive thirst like daily spontaneous drinking. Pharmacologic thirst and pharmacologic-induced hypohydration should become the focus of attention for clinicians and healthcare professionals in clinical settings. Pharmacologic thirst and pharmacologic-induced hypohydration have been reviewed comprehensively. Documented mechanisms of hydration status alteration by polypharmacy include decreased thirst sensation, diarrhea, increased urine volume, decreased appetite, increased sweat production, and central thermoregulatory affectation [6].

With aging, lean body mass and total body water decline. In aged individuals, many body functions, like cognition, mobility, and thirst sensation, decline [7]. On the other side of the coin, the aged are exposed to various health problems, such as, amongst others, metabolic syndrome and cardiovascular and chronic kidney diseases [8]. Johnson [9] suggested the cause-effect of low water intake in the aging process. It is plausible that improving water intake, to a significant extent, is a strategic interventional modality for healthy aging.

\section{Epidemiology of Fluid Intake Patterns}

Findings of various fluid consumption and its determinants using Liq.In7 records in 13 countries worldwide $[10,11]$ have enriched the science of hydration for health. The use of harmonized Liq.In 7 records in those countries has demonstrated its reputation in minimizing unnecessary data variability due to inconsistency in methodologies $[12,13]$.
Two articles in the present proceedings describe further analyses of fluid intake habits and patterns in children and adolescents $[14,15]$. Two findings can be emphasized: first, the finding that a high proportion of children and adolescents did not meet the European Food Safety's Adequate Intake recommendations for total fluid intake raised public health concerns about their hydration status and its related health and wellbeing outcomes; second, consumption of sugar-sweetened beverages (SSBs) in a significant proportion of children and adolescents, with low or high drinkers, raised the issue of future public health problems of noncommunicable diseases. The substantial contribution of SSBs consumption to daily sugar intake has raised concerns due to its adverse impacts on health [16]. SSBs consumption of adolescents at school strongly indicates the need to promote healthy drinking, aiming to prevent future unfavorable health outcomes and reduce the burden of noncommunicable diseases. Parental education holds its own importance. In many cases, responses to sweetness can originate from and form through repetitive exposure to foods and beverages prepared regularly at home, which are determined by family choices or parental preferences [17].

\section{Psychology of Thirst and Hydration}

Two articles in the present proceedings discuss perspectives of fluid intake, hydration, and mood changes, alertness, and drinking behavior using the COVID-19 lockdown model.

Yoder et al.'s [18] findings on the effect of hydration on self-reported thirst and alertness have added to the discourse on the importance of hydration status on several aspects of cognitive function. An earlier study by Pross et al. [19] with a different study design demonstrated that in individuals with high water intake, restricted water intake resulted in a significant increase in thirst and a decrease in contentedness, calmness, positive emotions, and vigor/activity. On the other hand, in those with low water intake, increased water consumption resulted in a significant decrease in fatigue/inertia, confusion/bewilderment, and thirst and a trend to lower sleepiness compared to baseline. Edmonds et al. [20] proposed that thirst moderates the effect of water, through centrally processing resources, on some aspects of cognitive performance. Their proposal was derived from their findings on the positive effects of water supplementation on the speed of responding, which may imply alertness. 
For 2 years, the COVID-19 pandemic has shown no signs of ending. Countries worldwide have implemented lockdown policies, and this policy has been implemented several times. Papies et al. [21] reported how drinking behavior changed during the UK lockdown. Water consumption during the lockdown did not increase. On the other hand, consumption of SSBs increased markedly, to the extent of negating the previous achievement of decreasing consumption of SSBs as part of a healthy lifestyle.

In their scoping review, Bennett et al. [22] reported that the effect of the COVID-19 lockdown both negatively and positively impacted dietary practices, and negative dietary habits were associated with other related poor lifestyles, including weight gain, mental health issues, and limited physical activity. These findings are of great concern given that unhealthy dietary behavior greatly affects the preexisting comorbidities [23] and the degree of severity of comorbid COVID-19 sufferers, which can aggravate inflammation and even increase the mortality rate [24].

\section{Hydration, Metabolic Responses, and Kidney Disease}

The previous proceedings have elaborated on the roles of the vasopressin system in the risk of diabetes and cardiorenal diseases $[25,26]$. Available data showed that increased water intake led to a decline in plasma osmolality and copeptin, a surrogate biomarker for vasopressin. High plasma copeptin is a significant independent predictor of the development of new-onset diabetes [26]. By using an acute experimental model, Seal et al. [27] demonstrated the importance of adequate water intake for plasma gluco-cortisol regulation. Arginine vasopressin (AVP as indicated by copeptin) induces hypersecretion of adrenocorticotropic hormone (ACTH) and cortisol, and this does not have a negative feedback loop [28]. Further comprehensive studies are needed to determine whether the acute effect of delayed reduction of plasma glucose was due to vasopressin or ACTH pathway.

There is another mechanism reported by Andres-Hernando et al. [29] concerning vasopressin. Animal studies have shown that dehydration causes stimulation of the polyol pathway in the hypothalamus, thereby increasing fructose and vasopressin production, leading to fructoseinduced obesity and metabolic syndrome. By increasing water intake in a fructose-fed mice model, they could demonstrate the reversal of obesity, which was associated with suppression of vasopressin levels.

Current Evidence in Water and Hydration Science
Two articles address the relations between hydration and kidney health. Lartey et al. [30] analyzed the NHANES epidemiological dataset from 3 sample years 2005/2006, $2007 / 2008$, and 2011/2012. The merged 3-period dataset revealed that participants with low total water intake were $\sim 7 \%$ more likely than their counterparts with high total water intake to develop moderate to severe kidney dysfunction.

Battistone et al.'s [31] original animal studies have opened new insights into the timely management of dehydration-induced acute kidney injury. In principle, there are three elements that exert their significant contributions to renal inflammation, namely type A intercalated cells, P2Y14 receptors, and UDP-glucose (UDPGlc) that are intertwined and mutually influencing each other, inducing an inflammatory cascade and, finally, leading to severe acute kidney injury, a potential medical complication with high mortality rate [32]. This study has raised a new hypothesis that blocking the UDP-Glc/ $\mathrm{P} 2 \mathrm{Y} 14$ pathway represents, therefore, a new therapeutic avenue for the prevention and or attenuation of dehydration-induced renal inflammation and dysfunction [33].

\section{Water, Hydration, Immune Response, and Gut Microbiota}

Universal and equitable access to safe and affordable drinking water is crucial for public health. Contaminated water and poor sanitation are linked to the transmission of preventable water-borne diseases, such as cholera, diarrhea, dysentery, hepatitis A, typhoid, and polio [34]. While the global burden of diarrheal disease remains high, continuous scientific interest in the effects of the drinking water microbiome and its sources and hydration status on the gut microbiota has attracted the attention of inter-disciplinary researchers $[35,36]$.

Vanhaecke [35] reported on the associations between the drinking water source and gut microbiota composition in the American Gut Project Database. Gut microbiota of 3,413 individuals was analyzed and categorized by the source of drinking water, namely bottled, tap, filtered, and well water. This study showed that drinking water source ranked among the key contributing factors explaining the gut microbiota variation both in alpha and beta diversity analyses, with effect sizes comparable to those of alcohol or diet type. Individuals drinking mostly well water also had higher fecal alpha diversity than the other groups. In addition, taxonomic differences were found in well water drinkers, with their potential of clin-

Ann Nutr Metab 2021;77(suppl 4):1-6 DOI: $10.1159 / 000521769$ 
ically important taxa, such as Bacteroides, Odoribacter, and Streptococcus, being depleted and Dorea being increased as compared to the other groups.

These findings show the importance of drinking water sources as a confounding factor in examining the human microbiome. Combined with the report of a previous study on the diversity of the microbiome across different habitats [37], this current report has enriched the evidence pointing to an ecosystem strategy for drinking water sources, adequate hydration, and gut health, and also their link with each other [38].

Willis et al. [36], the winner of this $\mathrm{H} 4 \mathrm{H}$ Early Career Researcher Award, reinforced the view of how hydration status affects the human fecal microbiota. This article presented three pathophysiological mechanisms that can lead to a clinical syndrome of gut dysfunction, including intestinal inflammation, gut barrier dysfunction, and fecal microbiota. Hydration status affects the dynamics of these three mechanisms. Two lessons can be learned from this study. First, the hypohydration state affects mucin production in the intestinal mucosal lining to the extent that the intestine becomes susceptible to inflammation $[39,40]$. Intestinal inflammation contributes to impairment of gut barrier function. This mechanism can be concluded by the negative correlation between copeptin (substitute biomarker for hydration status) and proteinbinding lipopolysaccharide. Second, there is a high plausibility that hydration status favors specific taxa of gut microbiota, which eventually contributes to its composition that favors the intestinal mucus barrier function.

The link between hydration and the immune system is an important future agenda in hydration for health [41, 42]. Innate and adaptive immunity play their parts in harmony and synergy against foreign and infectious agents. Eco-immunology research in reptiles has shown the role of hydration status on innate immunity using in vitro proxy parameters [43], and this phenomenon was not nutritional energy dependent. Many studies in exercise immunology showed the effect of strenuous exercise on the acute and chronic immune systems [44]. The immune system is very responsive to the workload of exercise. In individuals with intense exertion, several innate components, like natural killer cell activity and neutrophil oxidative burst activity, and adaptive components, like the $\mathrm{T}$ - and B-cell immune system, are functionally suppressed. At the same time, both plasma pro- and anti-inflammatory cytokines are elevated. Again, in this model of immune alteration, hydration status is not the only contributing factor; at the same time, energy balance, heat stress, and stress hormones make significant contributions [43, 45].

\section{Observational Remarks}

Sufficient scientific evidence on hydration for health in the present and previous proceedings confirmed the role of water and healthy hydration in a healthy life. We are faced with the question on how to monitor, evaluate, and appropriately correct our hydration status [46]. Monitoring and evaluating hydration status is important for athletes for their performance. However, there are strong relationships between hydration status and metabolic and kidney health; therefore, we also need to monitor it for our long-term health and well-being. One of the simplest methods is monitoring urine color [47]. The present proceedings have demonstrated the importance of measuring biochemical indicators, such as UDP-Glc, for early detection of organ disorders; an increase in UDP-Glc is an early sign of acute kidney injury due to dehydration.

Achieving adequate hydration in the elderly is a challenge in itself. Body composition changes markedly in the elderly by a decline in lean body mass and total body water and increased body fat mass. By virtue of physiological changes with aging, the level of euhydration in the elderly can differ from that of young adults. Multiple illnesses and polypharmacy often pose a higher risk for hypo- and de-hydration to the elderly than to young adults [48]. Declining brain health and prevailing neurodegenerative diseases [49] in the elderly, with their apparent neuron loss, may adversely affect the neural circuits controlling thirst. Therefore, it is vital not to forget healthy drinking practices in aged care in both community and institutional settings.

Epidemiological data regarding fluid consumption patterns in children and adolescents show that a reasonably high number among them are consuming SSBs. This situation constitutes a serious public health problem in the future. The World Health Organization (WHO) reported that the prevalence and incidence of type 2 diabetes remained high globally including in the developing countries [50]. Data are now accumulating demonstrating the relations between SSBs consumption and type 2 diabetes and cardiometabolic health [51], now including an association with early-onset colorectal cancer in women [52]. On the other hand, the development of the concept of sweetness in beverages will further affect the consumption patterns of SSBs in children and adolescents. The concept of sweetness was developed based on the understanding that sweetness in SSBs does not have to be due to sugar, but sugar can be replaced by sugar substitutes or low-calorie sweeteners. Switching sugar to sweet- 
eners still raises a new dilemma: what are the consequences of high low-calorie sweeteners exposure in children and adolescents, considering that children and adolescents prefer sweet tastes compared to adults $[53,54]$. The influence of parents on children and adolescents concerning the consumption of SSBs has raised the importance of household education on healthy drinking.

Findings regarding the role of water and hydration on the human gut microbiota deserve more attention. Two articles in the present proceedings provide strong evidence for the need for an ecological approach to water sources with the diversity of their microbiomes by their habitats. Many studies showed the importance of gut microbiota for better health outcomes, especially gastrointestinal health. An important lesson that we can learn from the two articles in this issue is that there is significant potential for orchestration between hydration, mucin production, and gut microbiota, which, if there is dysregulation of these three factors, may lead to inflammation, gut barrier dysfunction, and then, further negative implications for gut health.
Water is an essential element for biological function, and it is needed to achieve the required hydration status. The overall discussion points in the present proceedings have reinforced the value of hydration for health, which is fundamental but often ignored or forgotten and should be the focus of attention in many public-health interventions in both developed and developing countries.

\section{Conflict of Interest Statement}

W.L. has served as a Scientific Committee member of the Hydration for Health conference and received a consulting fee from Danone Research. He is also the Chair of the Indonesian Danone Institute Foundation and receives relevant honoraria.

\section{Funding Sources}

This work is supported by Danone Research.

\section{References}

1 Kavouras SA, Anastasiou CA. Water physiology: essentiality, metabolism, and health implications. Nutr Today. 2010;45(6S):S27-32.

2 Armstrong LE. Hydration Conference Spans Many Research Areas. Ann Nutr Metab. 2017;70(Suppl 1):1-3.

3 Armstrong LE, Kavouras SA. Thirst and drinking paradigms: evolution from single factor effects to brainwide dynamic networks. Nutrients. 2019 Nov;11(12):2864.

4 Bichet DG. New animal data on osmotic and hypovolemic thirst. Ann Nutr Metab. 2021;9(Suppl 4). doi: 10.1159/000520579.

5 Carroll HA. Redefining Thirst: Beyond dehydration and towards a holistic biopsychological model. Ann Nutr Metab. 2021;9(Suppl 4). doi: 10.1159/000520517.

6 Puga AM, Lopez-Oliva S, Trives C, Partearroyo T, Varela-Moreiras G. Effects of Drugs and Excipients on Hydration Status. Nutrients. 2019 Mar;11(3):669.

7 Morris JC. Is Alzheimer's disease inevitable with age?: lessons from clinicopathologic studies of healthy aging and very mild Alzheimer's disease. J Clin Invest. 1999 Nov;104(9):1171-3.

8 World Health Organization. World report on ageing and health. Geneva: World Health Organization; 2015.

9 Johnson EC. Hippocrates was right: Now what? Water as a part of healthy aging. Ann Nutr Metab. 2021;9(Suppl 4). doi: $10.1159 / 000520394$.
10 Ferreira-Pêgo C, Guelinckx I, Moreno LA, Kavouras SA, Gandy J, Martinez H, et al. Total fluid intake and its determinants: crosssectional surveys among adults in 13 countries worldwide. Eur J Nutr. 2015 Jun;54(Suppl 2):35-43.

11 Guelinckx I, Iglesia I, Bottin JH, De MiguelEtayo P, González-Gil EM, Salas-Salvadó J, et al. Intake of water and beverages of children and adolescents in 13 countries. Eur J Nutr. 2015 Jun;54(Suppl 2):69-79.

12 Gandy J. Water intake: validity of population assessment and recommendations. Eur J Nutr. 2015 Jun;54(Suppl 2):11-6.

13 Morin C, Gandy J, Brazeilles R, Moreno LA, Kavouras SA, Martinez H, et al. Fluid intake patterns of children and adolescents: results of six Liq.In7 national cross-sectional surveys. Eur J Nutr. 2018 Jun;57(Suppl 3):113-23.

14 Morin C, Gandy J, Moreno LA, Kavouras SA, Martinez H, Salas-Salvadaro J, et al. What characterizes fluid intake patterns across the world? Ann Nutr Metab. 2021;9(Suppl 4). doi: $10.1159 / 000520480$.

15 Iglesia-Altaba I, Miguel-Berges ML, Morin C, Moreno-Aznar LA. Fluid intake habits of Spanish children and adolescents: An update of the Liq.In7 survey. Ann Nutr Metab. 2021;9(Suppl 4). doi: 10.1159/000520516.

16 World Health Organization. Sugars intake for adults and children. Geneva: World Health Organization; 2015.
17 Pinard CA, Davy BM, Estabrooks PA. Beverage intake in low-income parent-child dyads. Eat Behav. 2011 Dec;12(4):313-6.

18 Yoder HA, Huffman AE, McCullough S, Johnson EC. Self-Reported Changes in Thirst and Alertness during Variable Prescribed Fluid Intake. Ann Nutr Metab. 2021;9(Suppl 4). doi: 10.1159/000521066.

19 Pross N, Demazières A, Girard N, Barnouin $\mathrm{R}$, Metzger D, Klein A, et al. Effects of changes in water intake on mood of high and low drinkers. PLoS One. 2014 Apr;9(4):e94754.

20 Edmonds CJ, Crombie R, Gardner MR. Subjective thirst moderates changes in speed of responding associated with water consumption. Front Hum Neurosci. 2013 Jul;7(363):363.

21 Papies EK, Rodger AK, Claassen MA, Lomann M. Recent Findings on the Psychology of Hydration Habits. Ann Nutr Metab. 2021;9(Suppl 4). doi: 10.1159/000520781.

22 Bennett G, Young E, Butler I, Coe S. The Impact of Lockdown During the COVID-19 Outbreak on Dietary Habits in Various Population Groups: A Scoping Review. Front Nutr. 2021 Mar;8:626432.

23 Ge E, Li Y, Wu S, Candido E, Wei X. Association of pre-existing comorbidities with mortality and disease severity among 167,500 individuals with COVID-19 in Canada: A population-based cohort study. PLoS One. 2021 Oct;16(10):e0258154. 
24 de Almeida-Pititto B, Dualib PM, Zajdenverg L, Dantas JR, de Souza FD, Rodacki M, et al.; Brazilian Diabetes Society Study Group (SBD). Severity and mortality of COVID 19 in patients with diabetes, hypertension and cardiovascular disease: a meta-analysis. Diabetol Metab Syndr. 2020 Aug;12(1):75.

25 Guelinckx I, Vecchio M, Perrier ET, Lemetais G. Fluid Intake and Vasopressin: Connecting the Dots. Ann Nutr Metab. 2016;68(Suppl 2):6-11.

26 Melander O. Vasopressin, from Regulator to Disease Predictor for Diabetes and Cardiometabolic Risk. Ann Nutr Metab. 2016;68(Suppl 2):24-8.

27 Seal A, Colburn AT, Suh HG, Kavouras SA. The acute effect of adequate water intake on glucose regulation in low drinkers. Ann Nutr Metab. 2021;9(Suppl 4). doi: 10.1159/000520479.

28 Enhörning S, Melander O. The Vasopressin System in the Risk of Diabetes and Cardiorenal Disease, and Hydration as a Potential Lifestyle Intervention. Ann Nutr Metab. 2018;72(Suppl 2):21-7.

29 Andres-Hernando A, Jensen TJ, Kuwabara M, Orlicky DJ, Cicerchi C, Li N, et al. Vasopressin mediates fructose-induced metabolic syndrome by activating the $\mathrm{V} 1 \mathrm{~b}$ receptor. JCI Insight. 2021 Jan;6(1):e140848.

30 Lartey D, Greenwood M, Linse G, Moyce S, Curl C, Spivak M, Johnson EC. Estimating differences in risk of chronic kidney disease based on water intake in a national sample. Ann Nutr Metab. 2021;9(Suppl 4). doi: 10.1159/000520666.

31 Battistone MA, Mendelsohn AC, Brown D, Breton S. Urinary UDP-glucose as a novel actionable biomarker of dehydration-induced acute kidney injury. Ann Nutr Metab. 2021;9(Suppl 4). doi: 10.1159/000520566.

32 Breton S, Battistone MA. Unexpected Participation of Intercalated Cells in Renal Inflammation and Acute Kidney Injury. Nephron. 2021 Oct:1-6.

33 Battistone MA, Mendelsohn AC, Spallanzani RG, Allegretti AS, Liberman RN, Sesma J, et al. Proinflammatory P2Y14 receptor inhibi- tion protects against ischemic acute kidney injury in mice. J Clin Invest. 2020 Jul;130(7): 3734-49.

34 Levy K. Does Poor Water Quality Cause Diarrheal Disease? Am J Trop Med Hyg. 2015 Nov;93(5):899-900.

35 Vanhaecke T. Associations between drinking water source and gut microbiota composition in the American Gut Project Database. Ann Nutr Metab. 2021;9(Suppl 4). doi: $10.1159 / 000520780$.

36 Willis NB, Muños CX, Mysonhimer AR, Edwards CG, Wolf PG, Hillman CH, et al. Hydration biomarkers are related to the differential abundance of fecal microbiota and plasma lipopolysaccharide binding protein in adults. Ann Nutr Metab. 2021;9(Suppl 4). doi: $10.1159 / 000520478$.

37 Walters KE, Martiny JB. Alpha-, beta-, and gamma-diversity of bacteria varies across habitats. PLoS One. 2020 Sep;15(9):e0233872.

38 Wahlqvist ML. Ecosystem Health Disorders - changing perspectives in clinical medicine and nutrition. Asia Pac J Clin Nutr. 2014; 23(1):1-15.

39 Redondo Useros N, Gheorghe A, Serrano Labajos R, Rebato E, Sanchez A. HYDRAGUT study: influence of HYDRAtion status on the GUT microbiota and their impact on the immune system. FASEB J. 2015;29(S1). https:// doi.org/10.1096/fasebj.29.1_supplement.593.1.

40 Paone P, Cani PD. Mucus barrier, mucins and gut microbiota: the expected slimy partners? Gut. 2020 Dec;69(12):2232-43.

41 Chabas D. Influence of suboptimal hydration on the immune response. Ann Nutr Metab. 2021;9(Suppl 4). doi: 10.1159/000520779.

42 Stookey JD, Allu PK, Chabas D, Pearce D, Lang F. Hypotheses about sub-optimal hydration in the weeks before coronavirus disease (COVID-19) as a risk factor for dying from COVID-19. Med Hypotheses. 2020 Nov; 144:110237.

43 Moeller KT, Butler MW, Denardo DF. The effect of hydration state and energy balance on innate immunity of a desert reptile. Front Zool. 2013 May;10(1):23.
44 Nieman DC, Wentz LM. The compelling link between physical activity and the body's defense system. I Sport Health Sci. 2019 May;8(3):201-17.

45 Mitchell JB, Dugas JP, McFarlin BK, Nelson MJ. Effect of exercise, heat stress, and hydration on immune cell number and function. Med Sci Sports Exerc. 2002 Dec;34(12):1941-50.

46 Anderson T, Adams WM, Wideman L. Modeling hydration status given daily measures of body mass, urine color, and thirst. Ann Nutr Metab. 2021;9(Suppl 4). doi: $10.1159 / 000520317$

47 Armstrong LE, Maresh CM, Castellani JW, Bergeron MF, Kenefick RW, LaGasse KE, et al. Urinary indices of hydration status. Int $J$ Sport Nutr. 1994 Sep;4(3):265-79.

48 Schols JM, De Groot CP, van der Cammen TJ, Olde Rikkert MG. Preventing and treating dehydration in the elderly during periods of illness and warm weather. J Nutr Health Aging. 2009 Feb;13(2):150-7.

49 Hardy J, Revesz T. The spread of neurodegenerative disease. $N$ Engl J Med. 2012 May;366(22):2126-8.

50 World Health Organization. Global report on diabetes. Geneva: World Health Organization; 2016.

51 Malik VS, Popkin BM, Bray GA, Després JP, Willett WC, Hu FB. Sugar-sweetened beverages and risk of metabolic syndrome and type 2 diabetes: a meta-analysis. Diabetes Care. 2010 Nov;33(11):2477-83

52 Hur J, Otegbeye E, Joh HK, Nimptsch K, Ng $\mathrm{K}$, Ogino S, et al. Sugar-sweetened beverage intake in adulthood and adolescence and risk of early-onset colorectal cancer among women. Gut. 2021 Dec;70(12):2330-6.

53 Fiorito LM, Marini M, Mitchell DC, Smiciklas-Wright H, Birch LL. Girls' early sweetened carbonated beverage intake predicts different patterns of beverage and nutrient intake across childhood and adolescence. J Am Diet Assoc. 2010 Apr;110(4):543-50.

54 Drewnowski A, Mennella JA, Johnson SL, Bellisle F. Sweetness and food preference. J Nutr. 2012 Jun;142(6):1142S-8S. 